Hydroécol. Appl. (1993) Tome 5 Vol. 2, pp. 71-96

\title{
Modélisation de la dégradation bactérienne de la matière organique. Application à la zone de turbidité maximale de l'estuaire de la Loire.
}

\author{
Modelling of degradation of organic matter by bacterial \\ populations. \\ Application to the zone of maximum turbidity of the Loire \\ estuary.
}

\section{Maurice}

Institut Français de Recherche pour l'Exploitation de la Mer, Direction de l'Environnement Littoral, B.P. 330, 83507 La Seyne/Mer, France.

Résumé. - La zone de turbidité maximale de l'estuaire de la Loire est sujette, en période estivale, à un important déficit en oxygène dissous qui peut atteindre l'anoxie en marée de vives eaux. Des campagnes de mesures ont été réalisées en septembre des années 1990 et 1991 sur toute la verticale dans cette zone, afin d'estimer la biodégradabilité de la matière organique et d'identifier les processus de biodégradation prenant part à l'anoxie.

Un modèle numérique a ensuite été mis en œuvre pour estimer la demande en oxygène dissous induite par les processus d'oxydation de la matière organique et par la nitrification.

Appliqué à la zone de turbidité maximale de l'estuaire de la Loire, les simulations ont été réalisées en prenant en compte les apports fluviaux en matière organique et en sels nutritifs. Les résultats permettent de comprendre et de quantifier le rôle de la crème de vase dans la demande en oxygène dissous du bouchon vaseux. En effet, la crème de vase est le siège de processus fermentatifs et d'hydrolyses. La formation de carbone organique dissous dans cette couche, estimée par le modèle en débit d'étiage, s'élève à un taux de $3 \mathrm{~g} \mathrm{C} \cdot \mathrm{m}^{-3}$.jour ${ }^{-1}$, identique au taux évalué à partir des mesures réalisées en septembre 1990 et 1991. La correspondance entre les résultats du modèle et des mesures est également vérifiée au niveau du taux de formation d'ammonium dans la crème de vase, soit en moyenne $6 \mathrm{nmol} \cdot \mathrm{g}^{-1} \cdot \mathrm{h}^{-1} \mathrm{~N}-\mathrm{NH}_{4}$, en période d'étiage estival. En 
débit d'étiage, il apparaît qu'à chaque période de vives eaux, soit en 7 jours, le substrat assimilé dans le bouchon vaseux provient pour $15 \%$ des produits organiques formés dans la crème de vase.

Mots-clés. - Estuaire de la Loire; zone de turbidité maximale; modèles chimique et biologique; demande en oxygène dissous; matière organique.

Abstract. - During the summer, the Loire estuary is subject to a very high deficit in dissolved oxygen, which may reach anoxia during the September spring tides. Measurement surveys were carried out in September 1990 and September 1991 throughout the maximum turbidity zone, in order to estimate the biodegradability of the organic matter and identify the processes of biodegradation involved in the anoxia.

A mathematical model was then developed to estimate the dissolved-oxygen demand resulting from degradation of the organic matter and nitrification of ammonia. The twolayer model represents the water column and the fluid mud.

Applying this model to the maximum turbidity zone in the Loire estuary, simulations were performed taking into account the inflow from the river in terms of organic matter and nutrient salts. The results obtained help in understanding and quantifying the influence of fluid mud on oxygen demand in the zone of maximum turbidity. Fluid mud is the center of processes of fermentation and hydrolysis. The model estimates formation of DOC (dissolved organic carbon) in this layer at $3 \mathrm{~g} \mathrm{C} \cdot \mathrm{m}^{3}$.day ${ }^{1}$ during neap tide low-flow periods, which corresponds to the rate estimated on the basis of measurements in September 1990 and 1991. The correlation between theoretical results and measurements has also been confirmed in terms of the rate of ammonia formation in the fluid mud: $6 \mathrm{nmol} \cdot \mathrm{g}^{1} \cdot \mathrm{h}^{-1} \mathrm{~N}-\mathrm{NH}_{4}$ on average during summer low-flow periods. When particles are suspended in the water column, the organic products formed by fermentation and anaerobic hydrolysis integrate into the aerobic metabolic cycle. The model shows that, at each 7-day spring tide during low-flow periods, $15 \%$ of the substrate assimilated in the zone of maximum turbidity is provided by fermentative organic products formed in the fluid mud.

Key words. - Loire estuary; maximum turbidity zone; chemical and biological models; dissolved oxygen demand; organic matter.

\section{INTRODUCTION}

Depuis plusieurs années, on observe dans l'estuaire de la Loire, une forte mortalité de mulets au cours de leur avalaison en septembre. Ce probleme est apparu directement lié à la présence d'une zone anoxique. Comme dans d'autres estuaires européens, les minima d'oxygène dissous correspondent à la zone de turbidité maximale (Morris et al., 1982). En Loire, en période d'étiage fort, cette zone peut remonter jusqu'à la commune de Nantes. Ifremer, à la demande du Port Autonome de Nantes Saint Nazaire, a développé plusieurs outils numériques en vue d'apporter des éléments de réponse au problème de l'anoxie. Un modèle d'oxygène dissous a été mis en œuvre afin de reproduire l'évolution des teneurs en oxygène dissous le long de l'estuaire (Thouvenin et al., 1992). Les premiers résultats ont souligné l'importance, dans la demande en oxygene dissous, du pro- 
cessus d'oxydation de la matière organique piégée dans le bouchon vaseux. Ce dernier point nous a conduit alors à développer un modèle mathématique reproduisant les principales étapes du cycle de dégradation de la matière organique dans la zone de turbidité maximale; c'est l'objet de cet article.

La zone d'étude comprend uniquement la zone de turbidité maximale de l'estuaire de la Loire (figure 1), qui en période de vives eaux peut se découper en deux couches: le bouchon vaseux et la crème de vase. Le bouchon vaseux (Glandeaud, 1938) où les concentrations en matières en suspension (MES) évoluent de $100 \mathrm{mg} \cdot \mathrm{l}^{-1}$ à $4 \mathrm{~g} \cdot l^{-1}$, résulte d'une accumulation de matériel particulaire au niveau du point nodal de l'estuaire, c'est-à-dire le lieu où les vitesses résiduelles du courant de marée et du courant fluvial s'annulent. En période de mortes eaux, une partie du matériel particulaire se dépose sous forme de crème de vase dont la teneur en matières en suspension peut atteindre $200 \mathrm{~g} \cdot \mathrm{I}^{-1}$. Nous avons constaté que cette couche est systématiquement anoxique. Le stock de matériel accumulé peut varier entre 500000 tonnes en étiage et 1000000 tonnes en crue (CSEEL, 1984). La fraction organique représente entre 3 et $10 \%$ de cette charge (Romaña et Thouvenin, 1987).

La mise en cuvre du modèle de dégradation de la matière organique dans la zone de turbidité de l'estuaire de la Loire a d'abord fait l'objet de recherches bibliographiques et de campagnes de mesures. Les résultats de ces recherches nous ont permis de poser les variables d'état et les pro-
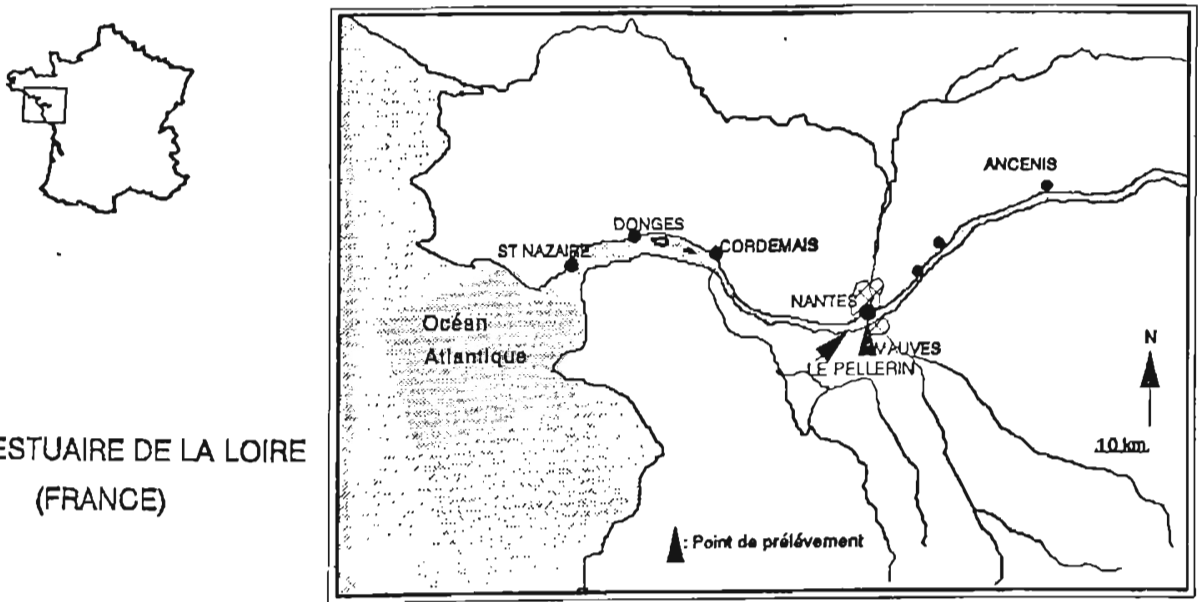

Fig. 1. - Présentation générale de l'estuaire de la Loire. Position des points de prélèvements des campagnes de 1990 et 1991.

Fig. 1. - Map of the Loire estuary. Sampling sites (September, 1990 and 1991). 
cessus modélisés, de caler les cinétiques, d'évaluer les conditions initiales ainsi que les apports fluviaux. Le modèle a ensuite été développé en accord avec les besoins du modèle d'oxygène dissous développé sur tout l'estuaire.

Si les précédentes campagnes de mesures réalisées sur l'estuaire apportent des informations intéressantes sur le fonctionnement global du système, elles sont insuffisantes pour appréhender les processus de minéralisation dans le compartiment "bouchon vaseux - crème de vase". C'est la raison pour laquelle nous avons organisé en septembre 1990 et 1991 des campagnes de mesures des principaux paramètres physico-chimiques, chimiques et microbiologiques non seulement dans la colonne d'eau, mais également dans la crème de vase où aucune mesure n'avait été réalisée auparavant. Les campagnes ont permis de mesurer non seulement les contenus en carbone et en azote de la matière organique, mais égaiement d'estimer la biodégradabilité de ses composés.

\section{MATÉRIEL ET MÉTHODES}

\section{Les campagnes du 27 septembre 1990 et du 17 au 23 septembre 1991} (Maurice, 1993) se sont déroulées au niveau d'épaisseur maximale de la lentille de vase recherché par échosondeur. Ce niveau a été détecté en 1990 à $3 \mathrm{~km}$ en aval de la commune de Nantes et en 1991 à $10 \mathrm{~km}$ en aval de cette même commune. Les prélèvements ont été effectués à chaque étal de pleine mer. La seconde campagne a été réalisée au cours d'une phase de remise en suspension de la crème de vase (tableau 1).

\subsection{Prélèvements}

En septembre 1990, les prélèvements ont été effectués dans la colonne d'eau à partir de bouteilles horizontales, à raison d'un tous les 1,50 mètre. Dans la crème de vase, ils ont été réalisés à partir d'un tube vertical en polyvinyl-chlorure de 3 mètres de hauteur et cloisonné tous les 25 centimètres. Cet appareil, conçu par nos soins, fût spécialement fabriqué à l'fremer. Chaque compartiment était muni d'orifices dont l'ouverture a été déclenchée dans la crème de vase après sa restabilisation; à la fin du remplissage, le tube fermé, fut remonté en surface.

En septembre 1991, les prélèvements ont été effectués à partir de bouteilles horizontales, aux profondeurs de $1,3,5,7,8$ et 9 mètres.

\subsection{Analyses des paramètres physico-chimiques, du carbone et de l'azote organique, de sels nutritifs, de la chlorophylle a et des phéopigments} A bord, les prélèvements ont aussitôt
été transvasés dans des flacons en
verre pour les mesures des paramè-
tres physico-chimiques (température,
salinité, oxygène dissous, pH et po- 
Tableau 1. - Caractéristiques hydrodynamiques et sédimentaires relevées aux points de prélèvements.

Table 1. - Hydrodynamic and sedimentary characteristics observed on sampling sites.

\begin{tabular}{|c|c|c|c|c|c|c|}
\hline \multirow{2}{*}{$\cdots$} & & \multirow{2}{*}{20.09 .91} & \multirow[b]{2}{*}{ 23.09.91 } \\
\hline & 27.09 .90 & 17.09 .91 & 18.09 .91 & 19.09.91 & & \\
\hline $\begin{array}{l}\text { Débits fluviaux } \\
\left(\mathrm{m}^{3} \cdot \mathrm{s}^{-1}\right)\end{array}$ & 300 & 126 & 130 & 135 & 136 & 115 \\
\hline \multicolumn{7}{|c|}{ Coefficlents de marée } \\
\hline & 31 & 28 & 29 & 37 & 49 & 83 \\
\hline \multicolumn{7}{|c|}{ Température eau de surface } \\
\hline$\left({ }^{\circ} \mathrm{C}\right)$ & 17 & 20 & 21 & 21 & 21 & 20 \\
\hline \multicolumn{7}{|l|}{ Profondeur totale } \\
\hline (m) & 10 & 10 & 10 & 10 & 10 & 10 \\
\hline \multicolumn{7}{|c|}{ Epaisseur de la crème de vase } \\
\hline $\begin{array}{l}(m) \\
\text { Etat de la surface }\end{array}$ & $\stackrel{2}{2}$ & $\begin{array}{l}3 \\
\text { calme }\end{array}$ & $\begin{array}{l}2,8 \\
\text { calme }\end{array}$ & $\begin{array}{l}2,5 \\
\text { calme }\end{array}$ & $\stackrel{2}{\text { calme }}$ & $\begin{array}{l}0,5 \\
\text { calme }\end{array}$ \\
\hline
\end{tabular}

tentiel d'oxydo-réduction, Eh) et les analyses de la matière organique dissoute (carbone organique dissous, COD et azote organique dissous, NOD). Des flacons en polyéthylène ont été utilisés pour les mesures des sels nutritifs, des MES, des matières volatiles en suspension (MVS) et du matériel organique particulaire (COP, NOP et pigments chlorophylliens totaux). Les flacons, maintenus à $4^{\circ} \mathrm{C}$, destinés à l'analyse des principaux paramètres physico-chimiques ont été transférés au laboratoire une heure après les prélèvements.

Les fractions dissoute et particulaire ont été séparées par filtration sur filtres Whatman GF/F, de porosité $0,45 \mu \mathrm{m}$, prépesés et traités au four à $450^{\circ} \mathrm{C}$ pendant 2 heures. L'eau destinée à la mesure du COD a été conservée en ampoule de $20 \mathrm{ml}$ scellée et traitée au persulfate de potassium. Les filtres, pour les analyses du matériel particulaire, ont été conservés au congélateur $\left(-25^{\circ} \mathrm{C}\right)$.

Dénombrements bactériens par épifluorescence

Les prélèvements des 17,19 et 23 septembre 1991 effectués à 3,5 et $9 \mathrm{~m}$ de profondeur ont été échantillonnés dans des flacons stériles de $10 \mathrm{ml}$, puis dilués au $1 / 10^{\mathrm{e}}$ et fixés avec $0,5 \mathrm{ml}$ de formol (à $25 \%$ ) pour $10 \mathrm{ml}$. Après traitement, la filtration a été réalisée à $0,2 \mu \mathrm{m}$ (filtres Nuclepores noirs, PC membrane $25 \mathrm{~mm}$ ). Des comptages bactériens par épifluorescence ont ainsi été réalisés sur des aliquotes formolées des échantillons placés dans le respiromètre. 


\subsection{Méthodes analytiques}

\section{Analyses chimiques et bactériologi-} ques

Les méthodes d'analyse des paramètres chimiques et bactériologiques sont présentées dans le tableau 2.

\subsection{Analyses des cinétiques de dégradation bactérienne}

Les mesures en continu de respiration bactérienne ont été réalisées sur les eaux brutes et interstitielles des 6 échantillons prélevés dans la crème de vase en septembre 1990; en 1991,

Tableau 2. - Méthodes analytiques utilisées sur les échantillons prélevés en septembre 1990 et 1991.

Table 2. - Analytical methodes used on samples taken in September 1990 and 1991.

Méthode d'analyse Références

Matières en suspension (totales et volatiles)

MES

Premier passage à l'étuve $\left(110^{\circ} \mathrm{C}\right.$ pendant $\left.6 \mathrm{~h}\right)$

MVS

Second passage à l'ètuve $\left(450^{\circ} \mathrm{C}\right.$ pendant $\left.6 \mathrm{~h}\right)$

élimination des matieres organiques

\section{Carbone et azote organique}

$\begin{array}{lll}\text { COD } & \begin{array}{l}\text { Oxydation à l'acide phosphorique } \\ \text { dosage du } \mathrm{CO}_{2} \text { par absorption IR } \\ \text { Oxydation du filtre; dosage du } \mathrm{CO}_{2}\end{array} & \text { Strickland et Parsons, } \\ \text { COP } & \begin{array}{l}\text { Analyse au } \mathrm{CHN} \text { (Carlo Erba) } \\ \text { Dosage par la méthode Kjeldahl }\end{array} \\ \text { NOD } & \begin{array}{l}\text { Soustraction de l'ammonium } \\ \text { Oxydation du filtre } \\ \text { NOP }\end{array} & \text { Analyse au CHN (Carlo Erba) }\end{array}$

\section{Pigments chlorophylliens}

Chlorophylle $a$ et phéopigments
Extraction des pigments par l'acétone (90\%) apres broyage des filtres

Spectrophotométrie
Lorenzen, 1967

\section{Sels nutritifs azotés}

Azote ammoniacal

Azote nitrique
Méthode au phénol-nitroprussiate + hypochlorite, Koroleff, 1969

Spectrophotométrie

Réduction sur colonne de cadmium traitée au Wood et al., 1967 cuivre,

Spectrophotométrie

\section{Biomasses bactériennes}

Bactéries totales
Traitement au $\mathrm{Na}_{4} \mathrm{P}_{2} \mathrm{O}_{7}$ : sonication ( $30 \mathrm{~s}$ sous $10 \mathrm{~W}$ ):

traitement au $\mathrm{N}_{3} \mathrm{Na}$ : dilution au $1 / 400$. coloration au DAPI $(0.5 \mu \mathrm{g} / \mathrm{ml})$ :

fixation à $4^{\circ} \mathrm{C}$ pendant $1 \mathrm{~h}$;

filtration à $0,2 \mu \mathrm{m}$ (filtres Nuclépores noirs;

comptage direct par épifluorescence méthode mise au point par

Maurice et Ferrara-

Guerrero

d'après Yoon et

Rosson, 1990 
elles ont été réalisées sur des échantillons issus des mêmes prélèvements que ceux destinés aux comptages bactériens.

\section{Description de l'expérience}

L'échantillon d'eau brute est placé dans un flacon stérile, plongé dans un bain-marie maintenu à la température de l'eau mesurée au moment du prélèvement. La quantité échantillonnée dans les flacons de $1250 \mathrm{ml}$, représente un gramme en poids sec. Elle est diluée avec de l'eau distillée et de l'eau de mer stérile en quantité suffisante pour reproduire la salinité du milieu. Ce flacon, placé à l'obscurité, est relié à un générateur d'oxygène dissous lui-même relié à un manomètre enregistrant en continu les dépressions liées à la consommation d'oxygène dans le milieu échantillonné. La teneur en oxygène dissous est ainsi maintenue à saturation tout au long de l'expérience. La consommation d'oxygène dissous est enregistrée en continu et cumulée sur le temps de la mesure (de 30 à 60 jours). Cette mesure a été réalisée sur des appareils délivrés par Voith $(\mathrm{GmbH}$, Allemagne) de type Sapromat D12.

\subsection{Estimation de la biodégradabilité de la matière organique : contrôles en début et fin d'expérience des teneurs en COP, NOP et en sels nutritifs}

Des mesures de COP, NOP, $\mathrm{NH}_{4}^{+}$, $\mathrm{NO}_{3}^{-}$et $\mathrm{NO}_{2}^{-}$ont été réalisées en début et en fin d'incubation, soit 60 jours, des échantillons de septembre 1991. La différence entre les concentrations en COP (ou NOP) mesurées en début et fin de respirométrie rapportée au carbone organique total (COT) ou à l'azote organique total (NOT), nous renseigne sur la biodégradabilité de la matière organique en supposant que les fractions carbonées (ou azotées) mesurées après 60 jours d'incubation sont lentement biodégradables voire réfractaires. Mais en réalité, ce pourcentage tend à majorer la fraction biodégradable du COP (ou NOP) puisque les mesures finales de COP (ou NOP) prennent en compte la matière organique formée pendant l'incubation des échantillons. Nous pouvons formuler plusieurs remarques quant aux résultats obtenus (Maurice, 1993) :

1. La biodégradabilité du COP dans la colonne d'eau augmente avec son enrichissement en populations bactériennes (de 11 à $24 \%$ du COP total).

2. La biodégradabilité du NOP dans l'eau reste à peu près constante sur les deux premiers jours de prélèvements et relativement élevé (en moyenne $48 \%$ du NOP total); ce qui laisserait supposer une dégradation préférentielle du NOP par rapport au COP. Cette hypothèse est à rapprocher de l'augmentation du rapport $\mathrm{C} / \mathrm{N}$ observé dans les dépôts.

3. La biodégradabilité du matériel organique particulaire de la crème de vase a tendance à diminuer avec le temps de séjour des particules dans cette couche ( 28 à $4 \%$ du COP). Ce résultat peut s'expliquer par une dégradation avancée de ce matériel au cours de son dépôt en environnement anoxique. 


\section{UTILISATION DES RÉSULTATS DES CAMPAGNES DANS LA MISE EN CEUVRE DU MODĖLE}

\subsection{Choix des processus}

Les résultats obtenus dans la crème de vase nous ont permis de mettre en évidence une formation de matière organique dissoute au cours des périodes de mortes eaux (figure 2); le taux de formation de carbone organique dissous (COD) a été estimé, à partir du temps de dépôt du COP, à $3 \mathrm{~g} \mathrm{C} \cdot \mathrm{m}^{-3} \cdot \mathrm{jour}^{-1}$. Cette couche est également le siège d'une ammonification et d'une dénitrification importantes; la production d'ammonium a pu être estimée à un taux compris entre 3 et $10 \mathrm{nmol} \mathrm{N}-\mathrm{NH}_{4} \cdot \mathrm{g}^{-1} \cdot \mathrm{h}^{-1}$ (figure 3).

\section{Septembre 1990}

Azote organique dissous (mg/l brut)

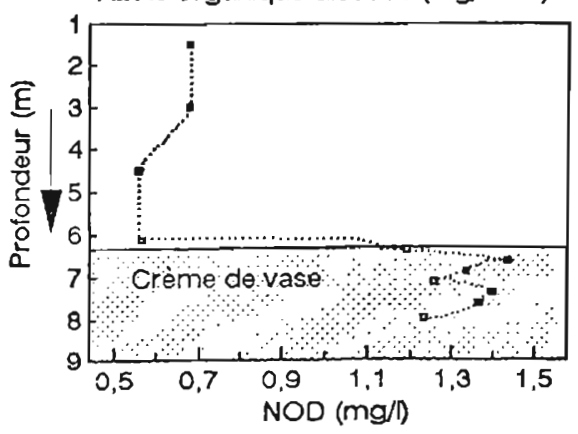

Septembre 1990

Carbone organique dissous ( $\mathrm{mg} / \mathrm{bnt}$ )

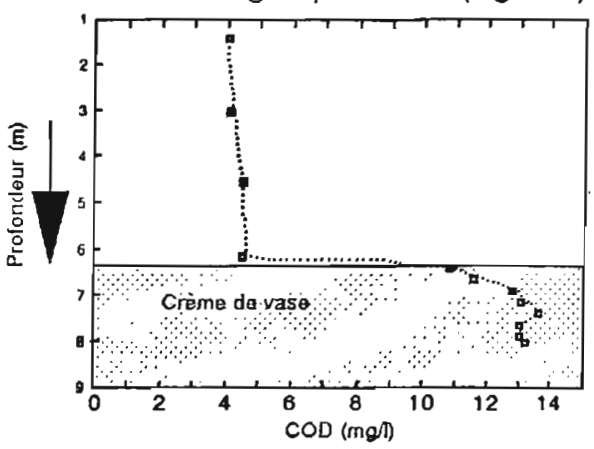

Fig. 2. - Profil vertical du carbone organique dissous mesuré dans la zone de turbidité maximale de l'estuaire de la Loire, septembre 1990 (marée de morte eau - débit d'étiage).

Fig. 2. - Vertical profile of dissolved organic carbon ( $\mathrm{DOC}$ in $\mathrm{mg} \mathrm{Cl}^{-1}$ ) measured in the zone of maximum turbidity of the Loire estuary (neap tide - low river flow).

Sels nutrittés azotés : $\mathrm{NH}_{4}^{+} \mathrm{NO}_{3}^{-}$et $\mathrm{NO}_{2}^{-}$

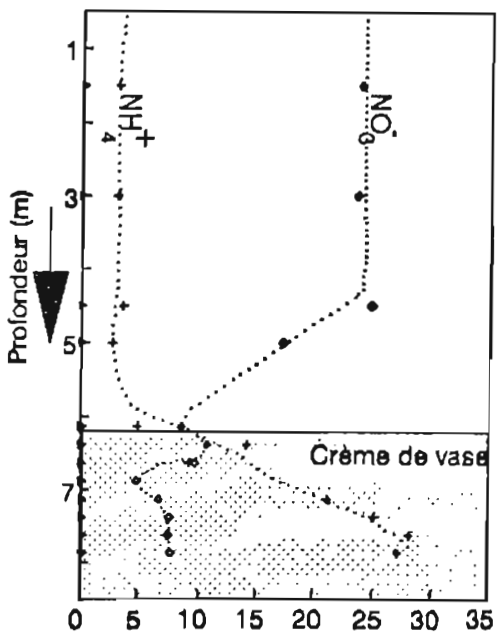

Figure 3. - Profils verticaux des composés azotés dissous mesurés en septembre 1990 dans la zone de turbidité maximale de l'estuaire de la Loire: (a) azote organique dissous $\left(m g \cdot l^{-1} \mathrm{~N}\right.$ ), (b) azote ammoniacal, nitrique et nitreux ( $\mu$ mol. $1^{\prime} \mathrm{N}$ ).

Figure 3. - Vertical profiles of dissolved nitrogenous compounds measured in September 1990 in the zone of maximum turbidity of the Loire estuary: (a) dissolved organic nitrogen (DON in $\mathrm{mg} \mathrm{N} \cdot \mathrm{I}^{-1}$ ), (b) ammonia $\left(\mathrm{NH}_{4}+\right.$ in $\left.\mu \mathrm{molN} \cdot \mathrm{I}^{-1}\right)$, nitrate $\left(\mathrm{NO}_{3}-\right.$ in $\left.\mu \mathrm{molN} \cdot \mathrm{I}^{-1}\right)$ and nitrite $\left(\mathrm{NO}_{2}-\right.$ in $\left.\mu \mathrm{mol} N \cdot 1^{-1}\right)$. 
Dans la colonne d'eau, nous nous sommes particulièrement intéressés à l'évolution du système au cours de l'érosion de la crème de vase. Le bouchon vaseux s'enrichit non seulement en matériel organique détritique mais également en biomasse bactérienne. Près de la surface, les teneurs en COD varient entre 4 et $5 \mathrm{mg} \cdot \mathrm{l}^{-1}$ entre le début et la fin de la vive eau. Cet enrichissement induit une augmentation de la demande en oxygène dissous du bouchon vaseux pouvant aller jusqu'à la désoxygénation.

Ces résultats nous ont conduit à considérer dans le modèle deux stocks de COD en fonction de leurs origines :

- un stock d'origine fluviale dont une majeure partie est lentement biodégradable au vu de son comportement conservatif observé le long de l'estuaire, probablement constitué en partie de substances humiques et

- un stock apporté par l'érosion de la crème de vase dont une partie est rapidement biodégradable.

En plus de l'évaluation des apports fluviaux, les informations recueillies au cours des campagnes nous ont conduit à poser différentes hypothèses au sujet du fonctionnement du modèle (tableau 3) :

\subsection{Evaluation des apports flu- viaux}

Les apports sont considérés dans le modèle en termes de flux entrant en amont de la zone de turbidité maximale; ceux issus de l'estuaire externe ont été négligés en raison de leur fai- ble importance par rapport aux apports de la zone fluviale. Ils ont été évalués à partir du produit des débits fluviaux et des concentrations des principaux composés chimiques constitutifs des variables modélisées (COD, COP, NOD, NOP, chlorophylle $a$, nitrate et ammonium) mesurées en 1988 à Ancenis (situé en zone fluviale).

Les hypothèses de répartition des apports en matière organique dans les variables d'état du modèle sont établies à partir des fractions biodégradables de la matière organique carbonée et azotée et utilisées dans le calcul des conditions initiales. II nous a paru intéressant de tenir compte, en particulier, des apports en composés d'origine algale puisque la Loire est connue pour son caractère eutrophe, avec des pics annuels de concentration en chlorophylle a supérieures à $200 \mu \mathrm{g} \cdot \mathrm{l}^{-1} \mathrm{Chl}_{a}$ (Crouzet, 1983). En effet, le matériel organique particulaire apporté par le fleuve peut être constitué soit, de phytoplancton ou de microorganismes morts n'ayant pas subi les premières étapes de dégradation, et seront considérés dans le modèle comme rapidement hydrolysables, soit de matériel détritique plus complexe, donc de dégradation plus lente. Le matériel phytoplanctonique, évalué à partir des concentrations en chlorophylle $a$, est supposé, pour sa fraction organique particulaire, rapidement hydrolysable. La concentration en carbone organique phytoplanctonique est calculée à partir du rapport entre le carbone organique phytoplanctonique et la chlo- 
Tableau 3. - Utilisation des résultats des campagnes dans la mise en œuvre du modèle. Table 3. - Use of measurement results in implementation of the model.

\section{Résultats}

\section{Dans la crème de vase}

- Formation de COD et de NOD (hydrolyse et/ou fermentation)

- Formation de $\mathrm{NH}_{4}$

- Utilisation de $\mathrm{NO}_{3}$

- Limitation de l'activité organotrophe par le niveau de dégradation de la matière organique
Modèle

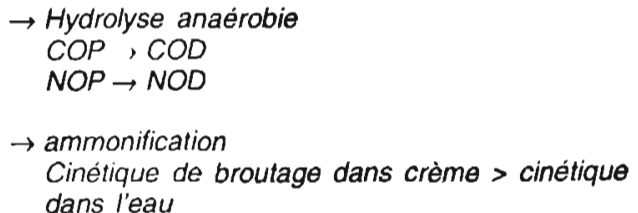

$\rightarrow$ respiration sur nitrates des bactéries hétérotrophes

$\rightarrow$ diminution du taux de croissance bactérienne en anaérobie

Dans la colonne d'eau, au cours de l'érosion de la crème de vase

- Enrichissement en matière organique détritique et vivante

- Biodégradabilité différente suivant origines de la m.o.

- Respirométries $\rightarrow$ Dilution des éléments de la crème de vase dans la colonne d'eau et forçage du particulaire par les MES

$\rightarrow$ Répartition des apports fluviaux dans modèle $=f(d i s /$ pant $, k, C / N)$

$\rightarrow$ Calage des cinétiques rophylle a; ce rapport est de 40 dans la zone fluviale de la Loire, valeur comparable à celle mesurée sur certains fleuves (Khalanski, 1984). Les résultats des campagnes réalisées en 1990 et 1991 montrent que le COP phytoplanctonique, défini par le carbone organique particulaire contenu dans la biomasse phytoplanctonique vivante, représente dans le bouchon vaseux seulement $10 \%$ du COP total; ces résultats confirment les observations des précédents auteurs (Saliot et al., 1984; Meybeck et al., 1988). Par ailleurs, nous pouvons remarquer dans la zone de turbidité maximale, la part prépondérante (80 à $95 \%$ ) de phéopigments (figure 4) dans les pigments totaux. Donc, si la quantité de matière organique particulaire apportée par le fleuve et piégée dans le bouchon vaseux est importante, en revanche, elle apparaît fortement dégradée. Cette hypothèse a également été émise au sujet du COD dont le comportement le long de l'estuaire est conservatif; le COD d'origine fluvial pourrait donc être constitué de composés humiques et fulviques lentement biodégradables. 
Septembre 1990

Chla, phéopigments el pigments totaux $(\mu / \mathrm{g})$

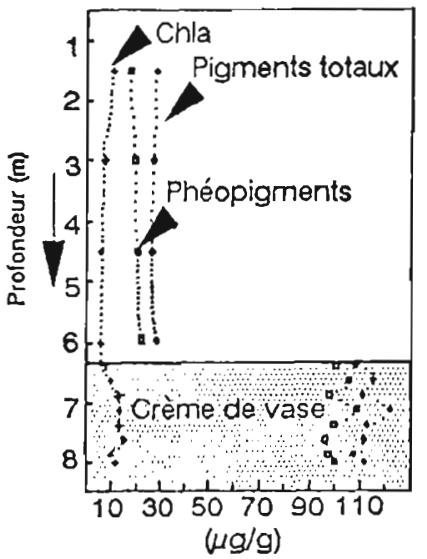

Figure 4. - Profils verticaux de la chlorophylle $a$, des phéopigments et des pigments totaux (chlorophylle a+phéopigments) mesurés dans la zone de turbidité maximale de l'estuaire de la Loire, en septembre 1990.

Figure 4. - Vertical profils of chlorophyll a, phaeopigments and total pigments (chlorophyll $a+$ phaeopigments) measured in the zone of maximum turbidity of the Loire estuary, September 1990.

Les apports fluviaux en matériel particulaire sont donc répartis dans le modèle selon trois origines (figure 5) :

\section{Origine phytoplanctonique}

Ces apports concernent les pigments chlorophylliens morts dont la dégradation n'a pas débuté.

COPphyto $\left(\mathrm{mg} \mathrm{C} \cdot \mathrm{I}^{-1}\right)=40 . \quad\left[\mathrm{Chl}_{a}\right]$ ( $\left.\mathrm{mg} \mathrm{Ch} \mathrm{Cl}_{a}\right|^{-1}$ )

$$
\text { NOPphyto }\left(\mathrm{mg} \mathrm{C} \cdot \mathrm{I}^{-1}\right)=\frac{\mathrm{COP} \text { phyto }}{5,6}
$$

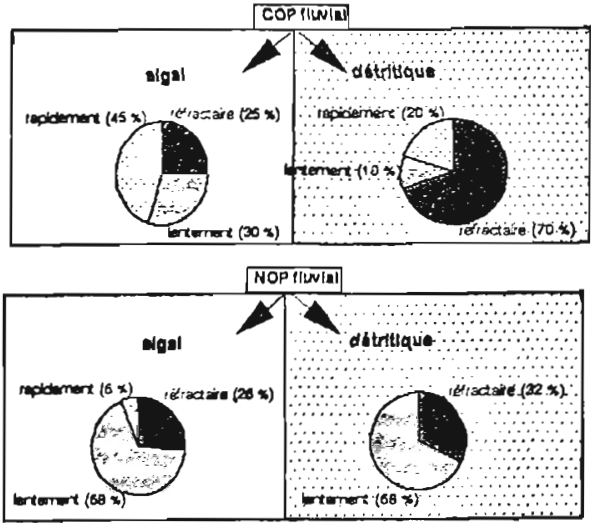

Figure 5. - Répartition des apports fluviaux en matériel organique dans les variables d'état du modèle.

Figure 5. - Distribution in the modelled compariments of organic matter supplied by inflow from the river.

\section{Origine détritique}

Le COP et le NOP d'origine détritique sont estimés à partir de la différence entre le matériel organique total et le matériel d'origine phytoplanctonique.

Les apports fluviaux sont répartis dans les variables d'état du modèle selon leur biodégradabilité (Servais, 1987; Otsuki et Hanya, 1972; Ogura, 1975; Billen, 1984; Tuschall et Brezonik, 1980):

\section{Origine anthropique}

Le flux de carbone organique apporté par les rejets urbains et industriels est estimé à partir du flux de $\mathrm{DBO}_{5}$ rejeté (Maurice, 1993), selon la relation stoechiométrique suivante:

Flux COT biodégradiable $=54 \%$. Flux $\mathrm{DBO}_{5}$ 
Ce flux est ensuite réparti entre les fractions dissoute et particulaire du carbone organique rejeté.

Les apports en sels azotés sont calculés à partir des valeurs d'azote ammoniacal et nitrique mesurée en 1988 à Ancenis (PANSN, 1988).

Les apports fluviaux en matériel organique dissous sont calculés à partir des concentrations en COD et NOD mesurées dans la zone fluviale et corrigées d'une coefficient représentatif de leur biodégradabilité; ils alimentent les fractions carbonée et azotée du substrat directement assimilable $\left(S_{d a}\right)$ et des substances rapidement hydrolysables $\left(D_{h r}\right)$; le détail des variables modélisées est explicité dans le chapitre suivant. Des expériences réalisées sur des échantillons d'eau prélevée en estuaire (Van Es et Laane, 1982) ou en rivière (Servais, 1987) ont permis de déterminer la fraction biodégradable du COD sur des temps d'incubation de 10 à 30 jours. Cette période correspond en effet au temps de séjour moyen de la matière organique dissoute dans l'estuaire; en débit d'étiage $\left(196 \mathrm{~m}^{3} \cdot \mathrm{s}^{-1}\right)$ par exemple, une tranche d'eau, partant d'Ancenis, met environ 24 jours pour atteindre Saint Nazaire (Salomon, 1976). La fraction directement assimilable peut être déterminée à partir de mesures d'acides aminés libres, de peptides et de protéines. Dans l'estuaire d'Ems Dollard par exemple, cette fraction paraît assez faible en été, d'environ $20 \%$ du COD total (Van Es et Laane, 1982). La fraction biodégradable du COD fluvial est donc estimée à $30 \%$, dont $20 \%$ est directement assimilable.

La part d'azote organique directement assimilable du NOD peut être approchée à partir du pourcentage d'acides aminés libres contenu dans les amines primaires (acides aminés libres et petites molécules peptidiques). Dans l'estuaire de l'Escaut (Billen, 1984), cette part s'élève à 30\%. D'autres auteurs (Tuschall et Brezonick, 1980) ont montré plus récemment qu'une part relativement importante de NOD (16 à 50\%), est constituée de polypeptides et de protéines dissoutes de plus haut poids moléculaires et donc hydrolysables. Nous avons donc choisi d'alimenter les concentrations des variables représentant l'azote organique dissous directement assimilable $\left(S_{\mathrm{daN}}\right)$ et hydrolysable $\left(D_{\mathrm{hrN}}\right)$ avec respectivement $30 \%$ et $20 \%$ de la concentration en NOD mesurée en Loire.

\section{MODĖLE DE DÉGRADATION DE LA MATIĖRE ORGANIQUE}

\subsection{Structure du modèle}

Le modèle de dégradation de la matière organique estime la demande en oxygène dissous induite par les processus d'oxydation de la matière organique et par la nitrification. Le modèle repose sur trois compartiments majeurs: la matière organique biodégradable, les biomasses bactériennes hétérotrophes et nitrifiantes et les sels nutritifs azotés.

L'originalité du modèle tient à la prise en compte simultanée des frac- 
tions carbonée et azotée de la matière organique. Cette dernière est découpée selon trois critères: sa phase dissoute ou particulaire, son rapport $\mathrm{C} / \mathrm{N}$ et sa cinétique moyenne de biodégradation. Quatre compartiments de matière organique sont modélisés, dont deux dissous : soit directement assimilable par les bactéries hétérotrophes, soit préalablement hydrolysables par des exoenzymes. La matière organique particulaire biodégradable est considérée soit lentement, soit rapidement hydrolysable (Henze et al., 196; Billen et Servais, 1988). Seul le processus d'assimilation directe conduit à une demande en oxygène dissous induite par la respiration des bactéries; la fraction anabolisée du substrat organique permet la formation de nouvelle biomasse bactérienne. Afin de considérer le plus justement possible les différents produits de la mortalité bactérienne dans les variables d'état, nous avons distingué la mortalité par lyse, de la mortalité par broutage. La première alimente les compartiments de matiere organique tandis que les produits d'excrétion des populations bactérivores enrichissent le milieu en ammonium. L'ammonium est ensuite incorporé par les populations bactériennes actives pour assurer leur biosynthèse. Dans la crème de vase anaérobie, les processus d'hydrolyse et de respiration sur nitrates sont modélisés.

La figure 6 représente schématiquement les variables et les processus biochimiques modélisés.

\subsection{Variables forçantes}

Les matières en suspension, la température et l'oxygène dissous sont considérés dans le modèle en tant que telles. Nous avons supposé que la matériel organique particulaire se dépose ou réintègre la colonne d'eau proportionnellement aux MES. Les concentrations en matières en suspension dans la colonne d'eau suivent des variations temporelles imposées par des cycles sinusoïdaux dont la période est de 14 jours (cycles lunaires); par contre, dans la crème de vase, la concentration des matières en suspension est supposée constante et égale à $100 \mathrm{~g} \cdot \cdot^{-1}$, valeur moyenne mesurée sur sa hauteur en 90 et 91 .

\subsection{Expressions mathématiques des processus modélisés}

\section{Assimilation directe, croissance et respiration bactérienne}

Les bases de calcul de l'assimilation directe reposent sur une formulation de type Michaëlis-Menten, liant l'activité à la biomasse bactérienne (Monod, 1949):

$$
\frac{d X}{d t}=\mu \cdot X
$$

avec, $\mu=\mu_{\max } \cdot \frac{\mathrm{S}}{\mathrm{K}_{\mathrm{s}}+} \dot{\mathrm{S}}$

$X$ : concentration de la biomasse bactérienne $\left(\mathrm{mg} \cdot \mathrm{l}^{-1} \mathrm{C}\right)$

$S$ : concentration en substrat assimilable $\left(\mathrm{mg} \cdot \mathrm{l}^{-1} \mathrm{C}\right)$

$\mu$ : taux de croissance bactérienne (jour $^{-1}$ ) 


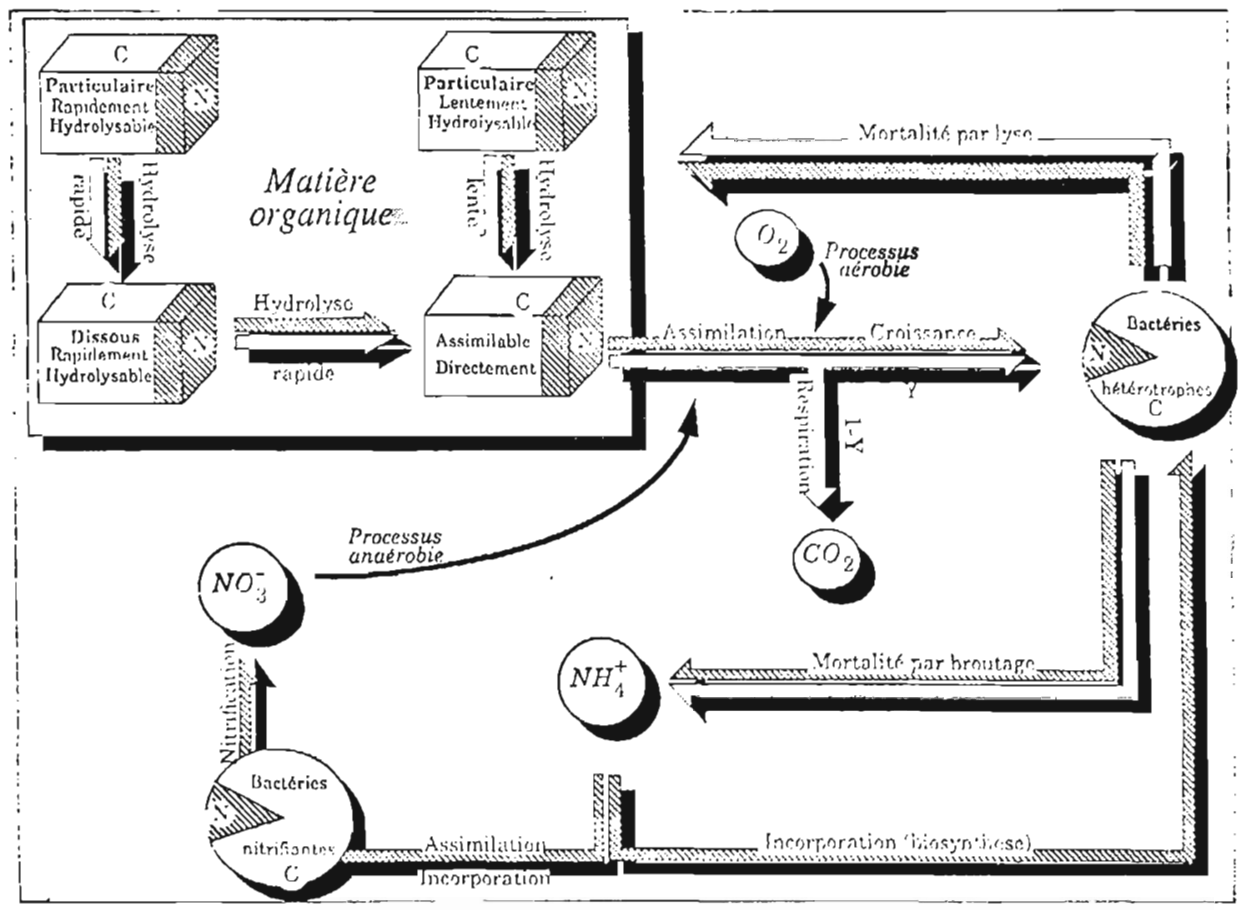

Figure 6. - Schématisation des processus modèlisés du cycle de dégradation bactérienne de la matière organique. Prise en compte des interactions avec les biomasses bactériennes hétérotrophes (hydrolyses et assimilation de la matière organique) et nitrifiante. L'ammonium constitue la source énergétique des populations bactériennes nitrifiantes : il est incorporé par les biomasses bactériennes pour leur biosynthese et excrété par les biomasses bactériennes et leurs prédateurs. Les nilrates, produits par nitrification, sont utilisés dans le processus de respiration bactérienne en anaérobiose. Figure 6. - Schematic of the modelled processes in the cycle of bacterial degradation or organic matter (broken down according to carbon and nitrogen content). Account is taken of interaction with heterotrophic and autotrophic bacterial biomass, hydrolysis, assimilation of organic matter and nitrification. Ammonia is the energy source for aerobic growth of the autotrophic biomass; release and uptake of ammonia by the bacterial biomass is shown. Nitrates produced by nitrification are taken up in the process of anaerobic bacterial respiration.

$\mu_{\max }$ : taux de croissance maximal (jour $^{-1}$ )

$\mathrm{K}_{\mathrm{s}}$ : constante à mi-saturation de la croissance bactérienne $\left(\mathrm{mg} \cdot \mathrm{l}^{-1} \mathrm{C}\right)$

A cette cinétique est appliquée une fonction d'atténuation en oxygène dissous $\left(S_{O D}\right)$ permettant de réduire ou d'annuler le processus en conditions anoxiques: $\mathrm{K}_{\mathrm{OD}}+\mathrm{S}_{\mathrm{OD}}$
Lorsque les teneurs en oxygène dissous sont insuffisantes, ce qui est le cas dans la crème de vase, la respiration bactérienne est réalisée sur les nitrates $\left(\mathrm{S}_{\mathrm{NO}}\right)$. La fonction d'atténuation permettant de prendre en compte cette éventualité est la suivante:

$$
\begin{array}{cc}
K_{O D}+S_{O D} & S_{N O} \\
K_{N O}+S_{N O}
\end{array}
$$


En conditions anoxiques, l'activité organotrophe est réduite de $30 \%$ par le terme stoechiométrique $\eta_{c a}$ placé en facteur du taux de croissance bactérienne (Maurice, 1993).

Une partie $(Y)$ de l'assimilation du substrat se rapporte au processus de croissance bactérienne; la partie complémentaire (1-Y) correspond au processus de respiration bactérienne qui permettra de calculer la demande en oxygène dissous.

\section{Hydrolyses}

Certains auteurs (Somville et Billen, 1983) ont montré que les vitesses d'hydrolyse de substrat artificiel prélevé en milieu marin, obéissent également à une cinétique de type Michaëlis-Menten. L'hydrolyse des compartiments organiques n'est pas limitée par une fonction d'atténuation en oxygène. Elle est donc également modélisée dans la crème de vase en référence au processus de fermentation observé en environnement anoxique (Marty et al., 1989).

\section{Mortalité bactérienne}

La mortalité bactérienne est considérée dans le modèle sous deux formes en fonction des produits issus de ce processus :

Une mortalité par lyse (bactérienne, virale, autolyse), dont les résidus (Fenchel et Jorgensen, 1977; Fenchel et Harrison, 1976) sont réintégrés en fonction de leurs constituants cellulaires, dans les compartiments de matière organique modélisés.

Une mortalité par broutage (Davis et Sieburth, 1984) : nous supposons que les composés formés au cours de cette mortalité correspondent aux produits d'excrétion des prédateurs bactériens (Van Wambeke et Bianchi, 1986; Sumi et Koike, 1990). Cette mortalité est donc prise en compte dans le modèle en terme de sources en ammonium. Nous avons considéré dans le modèle que $80 \%$ de la mortalité bactérienne était due au broutage; cette valeur a été confirmée au cours du calage des paramètres cinétiques mais n'a pas été évaluée in situ.

\section{Ammonification nette}

L'ammonification nette est définie par la différence entre l'ammonium formé par minéralisation de l'azote organique dissous et l'ammonium immobilisé pour la biosynthèse bactérienne. Nous supposons que les facteurs régissant la quantité d'ammonium libéré par les populations bactériennes sont le rendement d'assimilation du substrat $(\mathrm{Y})$ et la relation entre le rapport $C / N$ du substrat, $(C / N)_{s}$, et le rapport $\mathrm{C} / \mathrm{N}$ bactérien, $(\mathrm{C} / \mathrm{N})_{\mathrm{b}}$, (Lancelot et Billen 1985; Van Wambeke et Bianchi, 1986). L'expression mathématique de l'ammonification nette s'écrit alors :

$$
\begin{aligned}
& \mathrm{d}^{\mathrm{d}\left[\mathrm{N}^{*}-\mathrm{NH}_{4}\right]}=\left\{\begin{array}{c}
1 \\
\mathrm{Y}_{\mathrm{h}} \cdot(\mathrm{C} / \mathrm{N})_{\mathrm{s}} \\
-\mathrm{C} / \mathrm{N})_{\mathrm{b}}
\end{array}\right\} \quad \cdot \mu_{\mathrm{h}} \cdot \begin{array}{c}
\mathrm{S}_{\mathrm{da}} \\
\mathrm{K}_{\mathrm{s}}+\mathrm{S}_{\mathrm{da}}
\end{array} \cdot \mathrm{X}_{\mathrm{bh}} \\
& \begin{array}{c}
\text { assimilation } \\
\text { d'azote organique }
\end{array}
\end{aligned}
$$


$Y_{h}$ : rendement d'assimilation du substrat

$(\mathrm{C} / \mathrm{N})_{\mathrm{s}}$ : rapport $\mathrm{C} / \mathrm{N}$ du substrat $(\mathrm{C} / \mathrm{N})_{\mathrm{b}}$ : rapport $\mathrm{C} / \mathrm{N}$ bactérien

$\mathrm{S}_{\mathrm{da}}$ : concentration en substrat directement assimilable $\left(\mathrm{mg} \cdot{ }^{-1} \mathrm{C}\right)$

$\mu_{\mathrm{h}}$ : taux de croissance des bactéries hétérotrophes (jour ${ }^{-1}$ )

$\mathrm{K}_{\mathrm{s}}$ : constante à mi-saturation de la croissance bactérienne (mg. ${ }^{1{ }^{-1}} \mathrm{C}$ )

$X_{b h}$ : concentration en biomasse bactérienne hétérotrophe $\left(\mathrm{mg} \cdot \mathrm{l}^{-1} \mathrm{C}\right)$

Nitrification: croissance et respiration des bactéries nitrifiantes

Comme pour les processus exposés précédemment, nous avons choisi une loi de type Michaëlis - Menten pour calculer l'oxydation de l'azote ammoniacal en azote nitrique. Une fonction d'atténuation en oxygène dissous est également appliquée à ce processus. La croissance des bactéries nitrifiantes est calculée à partir de l'oxydation de l'ammonium et du rendement autotrophe. La part complémentaire du rendement autotrophe, exprimée en quantité d'oxygène consommée par quantité d'ammonium oxydée $\left(4,57-Y_{a}\right)$, permet de calculer la demande en oxygène dissous due à la respiration de cette biomasse.

La formulation mathématique utilisée dans le modèle est représentée sous forme matricielle en figure 7 . Aux colonnes sont attribuées les variables d'état et aux lignes, les processus.

Le modèle reproduit l'évolution temporelle des variables d'état dans la colonne d'eau et dans la crème de vase à partir de la résolution, en différences finies, des équations présen- tées dans la matrice de calcul. La méthode numérique utilisée procède par pas de temps fractionnaires (Usseglio-Polatera et Chenin-Mordojovick, 1988). Le pas de temps de calcul est de 2 minutes.

\subsection{Calages des paramètres cinétiques}

Les paramètres cinétiques du modèle ont été calés sur les mesures en continu de respiration bactérienne dans des gammes de valeurs référées en bibliographie. La mortalité par broutage n'a pas été considérée dans le calage à partir des respirométries effectuées sur les fractions dissoutes, où l'on suppose que les prédateurs bactériens ont été éliminés (Sherr et Sherr, 1983). Les produits des processus de nitrification et de minéralisation de la matière organique azotée, ont été vérifiés sur les valeurs de sels nutritifs mesurées en fin d'expérience. Le tableau 4 présente le jeu de paramètres cinétiques et de coefficients stoéchiométriques relatifs au meilleur calage.

\subsection{Vérification des résultats}

Les résultats du modèle ont été comparés aux mesures réalisées en juin et en septembre 1988 dans le bouchon vaseux. Les simulations ont débuté au $1^{\text {er }}$ janvier 1988; les apports fluviaux et la température ont été corrigés entre 3 et 4 fois par mois en fonction des débits, des concentrations et des températures mesurés 


\begin{tabular}{|c|c|c|c|c|c|c|c|c|c|c|c|c|c|c|c|}
\hline & 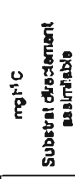 & $\frac{z}{2}$ & 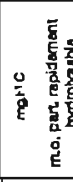 & 曾 & 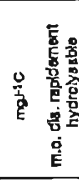 & $\frac{z}{2}$ & 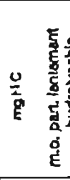 & 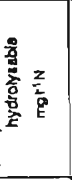 & 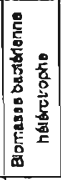 & 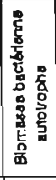 & 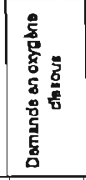 & 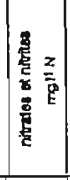 & 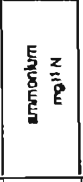 & & \\
\hline Compost 1 & $c^{s}$ & $\left.\right|^{\infty} N$ & $c{ }^{P_{h}}$ & $N$ & $c^{D_{r r}}$ & $N$ & $c{ }^{P_{H}}$ & in & $\begin{array}{c}X_{m} \\
c\end{array}$ & $\begin{array}{c}x_{2} \\
c\end{array}$ & $\begin{array}{l}\text { s.o } \\
\infty\end{array}$ & $\begin{array}{l}S_{m} \\
N\end{array}$ & $S_{N}$ & $\begin{array}{c}\text { Creigine } \\
\text { fous') }\end{array}$ & $\begin{array}{l}\text { Aaes } \\
\text { (moll') }\end{array}$ \\
\hline $\begin{array}{l}\text { Croissance } \\
\text { aćrobie } \\
\text { hótérotrophos } \\
\text { Incorporation } \\
\text { de NH4 }\end{array}$ & $\begin{array}{l}-\frac{1}{y_{h}} \times \\
\left(1-\frac{1)}{(C N)_{3}}\right.\end{array}$ & 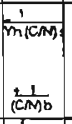 & & & & & & & 1 & & $\frac{1-Y h}{Y h}$ & & $\begin{array}{l}\frac{1}{m(C N) s} \\
-\frac{1}{(C M N)}\end{array}$ & $\begin{array}{l}\text { ph. } \frac{\text { Sda }}{\text { Kst } \overline{S d a}} \\
\frac{\text { Sod }}{\text { Koh+Sod }}\end{array}$ & Xbh \\
\hline \begin{tabular}{|l|} 
Croissance \\
anaérobio \\
incororation \\
de Nf44
\end{tabular} & 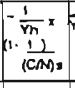 & 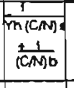 & & & & & & & 1 & & & $\frac{1-Y h}{\eta_{\operatorname{cs} Y h}}$ & $\frac{\frac{1}{1} \frac{1}{(C M) 2}}{\frac{1}{(C M) D}}$ & $\begin{array}{l}\text { Hh. Sda/(Ks +Sda } \\
\text { Koh/(Sod+Koh) } \\
\text {. Snol/Kno+Sno) }\end{array}$ & Xbh \\
\hline \begin{tabular}{|l|} 
Crgissance \\
aerobie \\
autotrophes \\
Incorporation \\
de NH4 \\
\end{tabular} & & & & & & & & & & 1 & $\frac{4,57-Y a}{Y a}$ & $\frac{1}{Y a}$ & $-\frac{1}{Y_{a}}$ & $\begin{array}{c}\mu \mathrm{a} \frac{\mathrm{Snh}}{\mathrm{Knh}+\mathrm{Snh}} \\
\frac{\text { Sod }}{\mathrm{Kog}+\text { Sod }} \\
\end{array}$ & Xoa \\
\hline \begin{tabular}{|l|} 
Mortalité \\
hétórotrophes
\end{tabular} & \begin{tabular}{|l|}
$a 212 n$ \\
$1-1$ \\
$(c m) b$ \\
\end{tabular} & $\begin{array}{l}0.212 \mathrm{n} \\
-\frac{1}{(\mathrm{CN}) \mathrm{b}} \\
\end{array}$ & 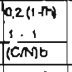 & $\frac{0.2(1+1)}{\frac{1}{(\mathrm{CN}) \mathrm{V}}}$ & & & 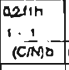 & \begin{tabular}{|c|}
$0.21 \mathrm{th}$ \\
1 \\
(CAM) \\
\end{tabular} & -1 & & & & $\frac{0,8}{(C, N) b}$ & $b_{h}$ & Xbh \\
\hline $\begin{array}{l}\text { Mortalité } \\
\text { autotrophos }\end{array}$ & $\begin{array}{l}0.122 \\
1 \frac{1}{(\mathrm{CNM}) \mathrm{D}}\end{array}$ & $\begin{array}{c}0.212 \mathrm{a} \\
1 \\
\text { (Cकाल) }\end{array}$ & \begin{tabular}{|l|}
$0.2(1+2)$ \\
$\frac{-1}{(\operatorname{con})^{b}}$ \\
\end{tabular} & $\frac{0.2(1+A)}{\frac{1}{(C M) b}}$ & & & \begin{tabular}{|c|}
$a, 2112$ \\
$1 \frac{1}{(\mathrm{CM}) \mathrm{b}}$ \\
\end{tabular} & $\begin{array}{c}0211 \mathrm{a} \\
1 \\
\mathrm{C} / \mathrm{N}) \mathrm{b}\end{array}$ & & -1 & & & $\frac{\mathrm{QB}}{(\mathrm{CN}) \mathrm{b}}$ & $b_{a}$ & Xba \\
\hline $\begin{array}{l}\text { Hydrolyse } \\
\text { rapide } 1\end{array}$ & & & $\begin{array}{l}1-1 \\
\text { (COMPt }\end{array}$ & $\frac{-1}{(\mathrm{CN})^{P_{t}}}$ & $1-\frac{1}{\text { ICNPP) }}$ & $\frac{1}{(\operatorname{CAN})^{P_{t}}}$ & & & & & & & & $\mathrm{~h}_{\mathrm{hr}} \frac{\mathrm{Phr}}{\mathrm{K}_{\mathrm{p}}+\mathrm{Phr}}$ & Xbh \\
\hline \begin{tabular}{|l} 
Hydrolyse \\
rapide 2
\end{tabular} & $1-\frac{1}{\cos (\mathrm{C})}$ & $\frac{1}{\cos \theta \alpha}$ & & & $=1-\frac{1}{(C) d}$ & $\frac{1}{\text { (CM) }}$ & & & & & & & & $k_{h r 2} \frac{\mathrm{Dhr}}{\mathrm{Kd}+\mathrm{Dhr}}$ & Xbh \\
\hline $\begin{array}{l}\text { Hydrolyse } \\
\text { lente }\end{array}$ & $1-\frac{1}{(C N) P 1}$ & $\frac{1}{(C M)^{P 9}}$ & & & & & $-1-\frac{1}{(\mathrm{CM}) \mathrm{PA}}$ & $\frac{-1}{(\mathrm{CM}) \mathrm{P}}$ & & & & & & $K_{h l} \frac{P h l}{K D+P h l}$ & Xbh \\
\hline
\end{tabular}

Figure 7. - Présentation de la matrice de calcul. Aux 13 premières colonnes correspondent les variables d'état modélisées; la $14^{\mathrm{e}}$ colonne présente les cinétiques utilisées et la $15^{\mathrm{e}}$ colonne, la variable d'état représentative de la biomasse bactérienne prenant part activement dans chacun des processus. Aux lignes sont attribués les processus modélisés.

Figure 7. - Presentation of the calculation matrix. The first 13 columns correspond to the modelled state variables; the 14 th, the kinetic coefficients, and the 15 th, the state variable representative of the bacterial biomass actively involved in each of the processes presented on each row.

en Loire fluviale. Seules les sorties par advection des éléments dissous ont été prises en compte; en effet, ne disposant d'aucune information quant à l'expulsion du matériel particulaire hors du bouchon vaseux, nous avons considéré qu'il restait piégé. Cette hypothèse tend à surestimer les ré- sultats en COP, NOP et biomasses bactériennes. En ce qui concerne la comparaison des résultats aux mesures de COP, NOP, COD et NOD, des pourcentages de biodégradabilité sont appliquées aux mesures à partir de valeurs référées en bibliographique ou des mesures réalisées dans le 
Tableau 4. - Paramètres cinétiques, coefficients stoechiométriques et variables d'état du modèle.

Table 4. - Kinetic parameters, stoichiometric coefficients and state variables of the model.

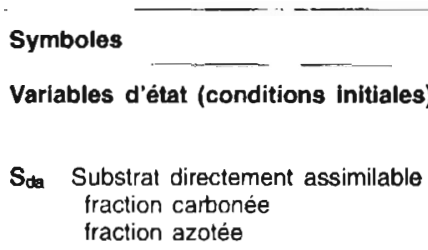

Dhr Matière organique dissoute rapidement hydrolysable fraction carbonée fraction azotee

$P_{h r}$ Matière organique particulaire rapidement hydrolysable fraction carbonée fraction azotée

$P_{\mathrm{h}}$ Matière organique particulaire lentement hydrolysable fraction carbonée fraction azotèe

$X_{\text {th }}$ Biomasse bactérienne hétérotrophe

$X_{\text {ba }}$ Biomasse bactérienne autotrophe

$\mathrm{C}_{\mathrm{NH}}$ Concentration en ammonium

$\mathrm{C}_{\mathrm{NO}}$ Concentration en nitrates

CMFs Concentration en matiere en suspension

\section{Paramètres cinétiques et constantes de Michaëlis - Menten (à $20^{\circ} \mathrm{C}$ )}

Un Taux de croissance maximal des bactéries hétérotrophes

$\mu_{\mathrm{a}} \quad$ Taux de croissance maximal des bactéries autotrophes

$\mathrm{K}_{5}$ Constante de Michaëlis de l'assimialtion du substrat direct

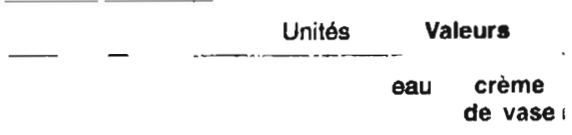

mg C. $\Gamma^{-1} \quad 0,88 \quad 1.04$

\begin{tabular}{|c|c|}
\hline $\begin{array}{l}m g C . I^{-1} \\
m g N . \Gamma^{1}\end{array}$ & $\begin{array}{l}0,44 \\
0,09\end{array}$ \\
\hline
\end{tabular}

$\begin{array}{lrr}\text { mg C. } \Gamma^{1} & 20,70 & 3861,00: \\ \text { mg N. } & 4,60 & 330,80\end{array}$

mg C. $\Gamma^{-1} \quad 62,00 \quad 5081.00:$

$\operatorname{mg~N.I^{-1}} 5,50 \quad 381.70$ :

mg C. $.^{-1} \quad 0.10 \quad 1,00$ :

mg C. $.^{-1} \quad 0,01 \quad 0,10$ :

$\operatorname{mg~N.I^{-1}} 0,07 \quad 0,38$ :

mg N.I ${ }^{1} \quad 0,31 \quad 0,10$ :

g.I $1,68 \quad 100,00$ !

$\mathrm{K}_{\text {rt. }}$ Constante de saturation en $\mathrm{O}_{2}$ de la croissance aérobie des bactéries hétérotrophes $\mathrm{mg} \mathrm{O}_{2} .^{1} \quad 0,25$

$\mathrm{K}_{n}$ Constante de saturation en $\mathrm{O}_{2}$ de la croissance aérobie des bactéries autotrophes $\mathrm{mg} \mathrm{O} .1^{-1} \quad 0,50$

bn Taux de mortalité spécifique des bactéries héterotrophes

$b_{\mathbf{a}} \quad$ Taux de mortalité spécifique des bactéries autotrophes

$\mathbf{k}_{\mathrm{hl}}$ Cinétıque d'hydrolyse du maténel organique lentement hydrolysable

$k_{r}$. Cinétıque d'hydrolyse du matérıel organıque rapıdement hydrolysable

$k_{h r 2}$ Cinétique d'hydrolyse des substances organiques rapidement hydrolysables

$K_{p l}$ Constante de Michaëlis de l'hydrolyse lente

$K_{\text {or }}$ Constante de Michaëlis de l'hydrolyse rapide du matériel particulaire

$K_{d}$ Constante de Michaëlis de lihydrolyse rapide des substances dissoutes

KNo Constante de saturation en nitrates

$\mathrm{K}_{\mathfrak{H} H}$ Constante de saturation en ammonium

\begin{tabular}{|c|c|}
\hline jour ' & 6,00 \\
\hline jour ${ }^{1}$ & 1,00 \\
\hline $\mathrm{mg} \mathrm{C.} \mathrm{I}^{-1}$ & 0,10 \\
\hline $\mathrm{mg} \mathrm{O}_{2 .} \mathrm{I}^{-1}$ & 0,25 \\
\hline $\mathrm{mg} \mathrm{O}_{2} 1^{-1}$ & 0,50 \\
\hline jour $^{-1}$ & 0,60 \\
\hline$j^{\prime} r^{-1}$ & 0,10 \\
\hline jour $^{-1}$ & 0,90 \\
\hline jour ${ }^{-1}$ & 1,00 \\
\hline jour & 2,00 \\
\hline $\mathrm{mg} \mathrm{C} . \mathrm{I}^{-1}$ & 2,00 \\
\hline $\mathrm{mg} \mathrm{C.} \mathrm{r}^{-1}$ & 3,00 \\
\hline $\mathrm{mg} \mathrm{C} . l^{-1}$ & 3,00 \\
\hline $\operatorname{mg~N} . \Gamma^{-1}$ & 5,00 \\
\hline $\operatorname{mgN} . \Gamma^{-1}$ & 2,00 \\
\hline
\end{tabular}

\section{Coefficients}

$\begin{array}{lr}Y_{h} \text { Rendement des bactéries hétérotrophes } & 0,30\end{array}$

$\begin{array}{ll}Y_{h} \text { Rendement des bactéries autotrophes } & 0,30\end{array}$

$\eta_{i}$ Facteur de correction de $\mu$. en conditions anoxiques $\quad 5,36$

$f_{01 \text { - }}$ Fraction particulaire lentement hydrolysable de la biomasse hétérotrophe lysée $\quad 0,05$

$f_{D 2^{n}}$ Fraction particulare directement assimilable de la biomasse hétérotrophe lysée $\quad 0,60$

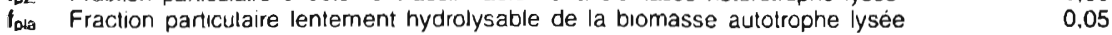

$\begin{array}{lll}f_{\text {Dza }} & \text { Fraction particulaire directement assimilable de la biomasse autotrophe lysée } & 0,60\end{array}$

$\begin{array}{lr}\text { any Fraction de la biomasse bacténenne lysée } & 0.20\end{array}$

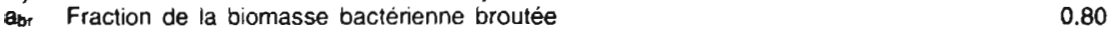


bouchon vaseux. La comparaison des résultats du modèle aux mesures est satisfaisante pour les éléments dissous mais d'autres points de mesures auraient été nécessaires pour assurer la validité du modèle en situation hydrodynamique critique et particulièrement des points de mesures régulières dans la crème de vase.

Par ailleurs, en ce qui concerne les taux de formation de COD et d'ammonium dans la crème de vase au cours de son dépôt en septembre 1991, on observe une bonne concordance entre les résultats du modèle et les estimations à partir des mesures.

\subsection{Etude de sensibilité}

Une étude rigoureuse de sensibilité du modèle a été réalisée pour des conditions hydrodynamiques de septembre 1988 (situation critique). La sensibilité est testée aux paramètres cinétiques, à la température, aux conditions initiales et aux apports (hypothèses de biodégradabilité). Le modèle apparaît particulièrement sensible aux conditions initiales en COP et NOP lentement hydrolysables. L'oxydation de ce matériel organique intervient dans la demande en oxygène dissous du bouchon vaseux après seulement trois mois de piégeage. Ainsi, en septembre, la demande en oxygène relative à une partie de la matière organique particulaire devrait concerner les apports de juin. En revanche, le modèle apparaît insensible aux conditions initiales des autres variables d'état ainsi qu'aux hypothèses de biodégradabilité de la matière organique d'origine fluviale.

En ce qui concerne les rejets urbains et industriels, seulement 3\% du carbone organique assimilé dans le bouchon vaseux, sur un cycle lunaire, est d'origine anthropique. Mais entre une situation avec prise en compte de ce type d'apports et une situation sans, la demande en oxygène dissous augmente de 12 à $15 \%$ si la fraction dissoute de la matière organique apportée par les rejets passe de 0 à $100 \%$, ce, pour un flux de 19 tonnes C.jour ${ }^{-1}$ (Maurice, 1933).

\section{RÉSULTATS}

Les simulations ont été réalisées conformément à deux situations : celle de juin et celle de septembre 1988, généralement caractérisée par une anoxie. Les concentrations initiales en matière organique et en sels nutritifs ont été calculées à partir des mesures de ces variables en ces mêmes mois. En juin et en septembre 1988 , les volumes d'eau moyens sont respectivement de $238 \cdot 10^{6} \mathrm{~m}^{3}$ et de $95 \cdot 10^{6} \mathrm{~m}^{3}$; la profondeur moyenne du bouchon vaseux est respectivement de 6 et $7 \mathrm{~m}$.

Les simulations ont été réalisées sur un cycle lunaire. La période d'un cycle lunaire peut être découpée en deux en fonction des coefficients de marée : nous supposons qu'à chaque marée de mortes eaux, soit 7 jours, correspond une phase de dépôt du 
matériel particulaire et qu'à chaque marée de vives eaux correspond une phase de remise en suspension de la couche crème de vase.

\section{Rôle de la crème de vase dans le fonctionnement biochimique du bouchon vaseux}

Les premiers résultats permettent de relativiser les sources de substrat organique à l'origine de la demande en oxygène dissous du bouchon vaseux; ils laissent apparaitre l'importance du rôle de la crème de vase dans le fonctionnement biogéochimique du système.

Sur un cycle de dépôt, soit 7 jours, la formation de COD dans la crème de vase, estimée par le modèle en débit d'étiage, s'élève à un taux de $3 \mathrm{gC} \cdot \mathrm{m}^{-3}$.jour ${ }^{-1}$, identique aux taux évalué à partir des mesures de sep. tembre 1990 et 1991. Les produits organiques des hydrolyses anaérobies enrichissent la crème de vase en substrat assimilable par les bactéries hétérotrophes. La remise en suspension de la crème de vase induit une augmentation de la charge organique biodégradable dans le bouchon vaseux. Au moment des marées de vives eaux, ce substrat entre dans le cycle de respiration aérobie en cours dans la colonne d'eau et contribue ainsi à augmenter le déficit en oxygène dissous. Le modèle considère en effet, que la biomasse bactérienne hétérotrophe dont l'activité est ralentie en environnement anoxique (crème de vase) est capable de reprendre un métabolisme aérobie si les conditions en oxygène dissous le permettent. A l'échelle d'un cycle lunaire, les résultats des simulations montrent que $65 \%$ de la demande en oxygène dissous est réalisée pendant la phase de remise en suspension de la crème de vase. En débit d'étiage, il apparaît qu'à chaque marée de vives eaux, le substrat assimilé dans le bouchon vaseux provient à $15 \%$ des produits formés dans la crème de vase (figure 8 ). En débit moyen, cette participation est d'environ $11 \%$ (figure 8 ).

En débit d'étiage, la demande en oxygène dissous du bouchon vaseux due au processus d'oxydation de la matière organique s'élève en moyenne à un taux de $4 \mathrm{~g} \mathrm{O}_{2} \cdot \mathrm{m}^{-3}$.jour ${ }^{-1}$. En raison des teneurs en ammonium non limitantes et des températures élevées, la nitrification augmente cette demande d'au maximum $20 \%$. La formation d'ammonium par ammonification et excrétion des population hétérotrophes, mise en évidence dans la crème de vase, permet en effet d'enrichir le bouchon vaseux en azote ammoniacal à chaque marée de vives eaux. Les mesures réalisées en septembre 1990 et 1991 ont permis d'estimer un taux de formation d'ammonium dans la crème de vase d'environ compris entre 3 et $10 \mathrm{nmol} \cdot \mathrm{g}^{-1} \cdot \mathrm{h}^{-1} \mathrm{~N}-\mathrm{NH}_{4}$. Le taux estimé par le modèle s'élève à $6 \mathrm{nmol} \cdot \mathrm{g}^{-1} \cdot \mathrm{h}^{-1} \mathrm{~N}-\mathrm{NH}_{4}$.

Par ailleurs, l'importante quantité de matière organique biodégradable dans la zone de turbidité maximale permet d'expliquer les taux élevés d'activité organotrophe. Ce taux estimé par le modèle en situation hydro- 
dynamique de septembre, atteint en moyenne $12 \mathrm{~g} \mathrm{C}^{-2} \mathrm{~m}^{-2}$.jour ${ }^{-1}$. A la fin du bloom phytoplanctonique fluvial et après les crues, la zone de turbidité maximale s'enrichit en matériel organique lentement biodégradable.

\section{CONCLUSION ET PERSPECTIVES}

La démarche développée dans cet article, vise à conjuguer les connaissances acquises depuis ces dernières années sur les processus régissant les interactions entre la matière organique biodégradable et les biomasses bactériennes, avec la modélisation mathématique. Cette démarche, appliquée à la problématique de l'estuaire de la Loire, a permis de développer un modèle mathématique estimant la demande en oxygène dissous d'une zone caractéristique de certains systèmes estuariens : la zone de turbidité maximale. L'exploitation de ce modèle permet dans une première étape, de comprendre le fonctionnement biogéochimique du système modélisé; dans une seconde étape, au-delà de l'aspect descriptif, d'estimer l'importance relative des différentes sources de matière organique piégée dans le bouchon vaseux intervenant dans le déficit en oxygène dissous.

\section{Situation de septembre 1988 _ Débit d'étiage}

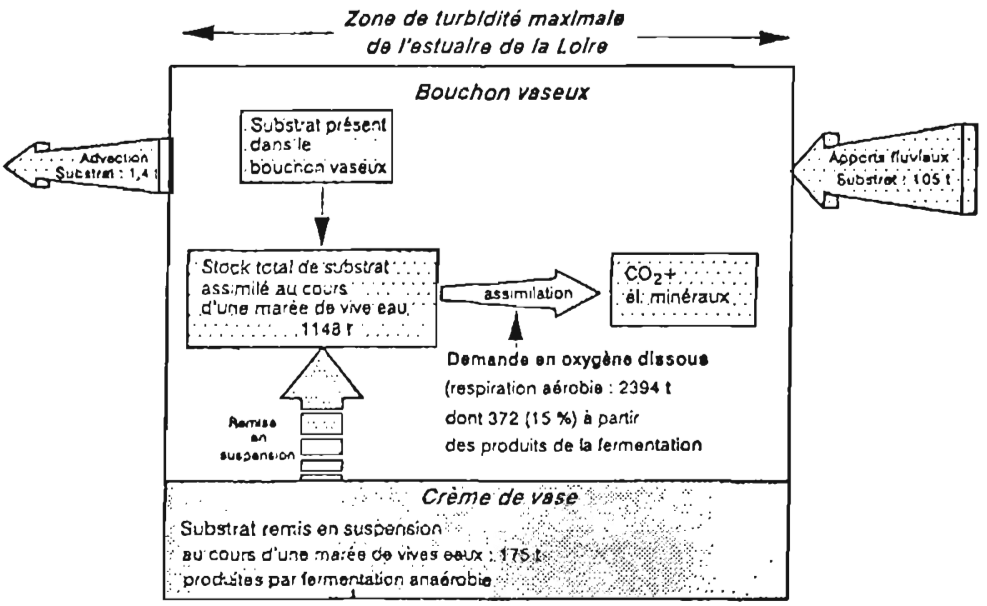

Figure 8. - Approche d'un bilan en substrat directement assimilable dans la zone de turbidité maximale, dans le cas d'un débit fluvial d'étiage $\left(226 \mathrm{~m}^{3} \cdot \mathrm{s}^{-1}\right)$. Les quantités en tonnes sont calculées sur 7 jours de marée de vives eaux, correspondant à la phase de remise en suspension de la crème de vase.

Figure 8. - Approximate budget of the directly assimilable substrate in the zone of maximum turbidity in a typical September situation of low river flow $\left(226 \mathrm{~m}^{3} \cdot \mathrm{s}^{-1}\right)$. Quantities expressed in tons are calculated over 7 days of spring tide, corresponding to the resuspension phase of fluid mud. 
Les résultats confirment le rôle majeur de la crème de vase dans la demande en oxygène dissous du bouchon vaseux. II apparaît qu'à chaque marée de vives eaux, le substrat assimilé dans le bouchon vaseux provient à $15 \%$ des produits formés dans la crème de vase.

L'importante quantité de matière organique biodégradable dans cette zone permet d'expliquer les taux élevés d'activité organotrophe, qui atteindrait en moyenne $12 \mathrm{~g} \mathrm{C} \cdot \mathrm{m}^{-2}$.jour ${ }^{-1}$; mais en revanche, la présence de substances organiques déjà fortement dégradées (substances humiques ou fulviques) semble limiter le développement bactérien.

En ce qui concerne l'impact des effluents dans l'activité organotrophe, en débit d'étiage, moins de $3 \%$ du COD assimilé est d'origine anthropique. En revanche, les apports urbains et industriels peuvent augmenter la demande en oxygène dissous d'au maximum $15 \%$.

Ces résultats mettent en évidence l'importance de l'activité organotrophe dans le fonctionnement du systeme "bouchon vaseux - crème de vase". Cependant, le modèle présenté dans cet article est basé sur des hypothèses dont certaines méritent d'être affinées. Une meilleure connaissance des cinétiques de biodégradabilité de la matiere organique en général, et plus particulierement de celle d'origine phytoplanctonique serait appréciable. Des informations manquent encore sur la réponse du développement bactérien aux variations des conditions en oxygène du milieu.
L'application principale de cette étude contribue à l'amélioration du modèle global d'oxygène dissous dans l'estuaire de la Loire (Thouvenin et al., 1992). En effet, la seconde version de ce modèle permet de décrire plus en détails la formation, l'évolution et l'origine de la matière organique piégée dans le bouchon vaseux. L'exploitation de ce dernier modèle nécessite donc une meilleure définition des stocks de matière organique arrivant de l'amont ainsi que de ceux issus de la remise en suspension de la crème de vase. La classification de la matière organique selon sa biodégradabilité et ses cinétiques de dégradation ont été transposées à cette nouvelle version du modèle d'oxygène dissous. Associées à la prise en compte d'une formation de produits organiques dissous rapidement biodégradables dans la crème de vase, ces hypothèses supplémentaires permettent de mieux ajuster les résultats du modèle aux mesure d'oxygène dissous.

\section{REMERCIEMENTS}

Ces recherches ont été menées au sein d'lfremer sous la direction de L.A. Romaña (Centres de La Seyne Sur Mer, de Brest et de Nantes. France), à partir du financement de l'Association pour la Protection de l'Environnement de l'Estuaire de la Loire (APEEL). Nous tenons à remercier toutes les personnes qui ont participé à cette étude, en particulier le personnel du Port Autonome de NantesSaint Nazaire pour sa participation aux campagnes, et du Laboratoire de Micro- 
biologie Marine de Luminy ( $M$. Bianchi, CNRS-UPR 223, Marseille, France) pour son accueil et sa collaboration à la mise en œuvre d'une méthodologie de comptages bactériens en milieu fortement chargé en particules.

\section{BIBI_IOGRAPHIE}

Billen G., 1984. Heterotrophic utilization and regeneration of nitrogen, in: $\mathrm{He}$ terotrophic activity in the sea, edited by Hobbie J.E. et Williams P.J. Le B., Vol. 15, 313-355.

Billen G. et Servais P., 1988. Modélisation des processus de dégradation bactérienne de la matière organique en milieu aquatique, In: Microorganismes dans les écosystèmes océaniques, edited by Bianchi, Masson, Paris, 219245.

Crouzet P., 1983. L'eutrophisation de la Loire. Water Supply, 1, 131-144.

CSEEL, 1984. Rapport final du Comité Scientifique pour l'Environnement de l'Estuaire de la Loire. Rapport CNEXO/CNRS, $n^{\circ} 55$.

Davis P.G. et Sieburth J.Mc. N., 1984. Estuarine and oceanic microflagellate predation of actively growing bacteria: estimation by frequency of dividing-divided bacteria. Mar. Ecol. Progr. Ser., 19, 237-246.

Fenchel T. et Harrison P., 1976. The significance of bacterial grazing and mineral cycling for the decomposition of particulate detritus. In: The role of terrestrial and aquatic organisms in decomposition processes, edited by Blackwell, Anderson J/.M., Oxford, 285-299.

Fenchel T.M. et Jorgensen B.B., 1977. Detritus food chains of aquatic ecosystems: the rule of bacteria, in: Advances in Microbiol Ecology, Vol. I, edited by Alexander M., Plenum Press, New York, 1-58.

Glangeaud L., 1938. Transport et sédimentation dans l'estuaire et à l'embouchure de la Gironde. Bull. Soc. Géol de $F_{r}$, $5^{\mathrm{e}}$ série, 8, 599-631.

Henze M., Grady C.P.L., Gujet W., Marais G.v.R. et Matsuo T., 1986. Activated Sludge Model $N^{\circ} 1$, Scientific and Technical Reports $N^{\circ} 1$, par l'International Association on Water Pollution Research and Control, IAWPRC.

Khalanski M., 1984. Evaluation de la biomasse phytoplanctonique des eaux courantes par le dosage de la chlorophylle. Verh. Internat. Verein. Limnol., 22: 2010-2019.

Koroleff F., 1969. Direct determination of ammonia in natural waters as indophenol blue. Hydr. Comm., ICES, C.M. 1969/C, 9.

Lancelot C. et Billen G., 1985. Carbon Nitrogen relationships in nutrient metabolism of coastal marine ecosystems. Adv. in Aquatic Microbiol., Vol. 3 , 263-321.

Lorenzen C.J., 1967. Determination of chlorophyll and phaeopigments: spectrophotometric equations. Limnol. Oceanogr. Lawrence Can., 6, 343-346.

Marty D., Bertrand J.C. et Caumette P., 1989. Les métabolismes bactériens dans les systèmes sédimentaires marins, in: Microorganismes dans les écosystèmes aquatiques, edited by Masson, Paris, 101-151.

Maurice L., 1993. Modélisation du cycle de dégradation bactérienne de la matière organique. Application à la zone de turbidité maximale de l'estuaire de la Loire. Thèse de $3^{e}$ cycle. Institut National Polytechnique de Toulouse.

Meybeck M., Cauwet G., Dessery S., Somville M., Gouleau D. et Billen G., 1988. Nutrients (organic C, P, N, Si) in the eutrophic river Loire (France) and its estuary. Estuar., Coast. and Shelf Sci., 27, 595-624. 
Monod J., 1949. The growth of bacterial cultures. Ann. Rev. Microbiol., 3, 371394.

Morris A.W., Loring D.H., Bale A.J., Howland R.J.M., Mantoura R.F.C. et Woodward E.M.S., 1982. Particles dynamics, particulate carbon and the oxygen minimum in an estuary. Oceanol. Acta, Vol. $5, n^{\circ} 3$.

Newell R.C. et Linley E.A.S., 1984. Significance of microheterotrophs in the decomposition of phytoplancton : estimates of carbon and nitrogen flow based on the biomass of plankton communities, Mar. Ecol. progr. Ser., 16, 105-119.

Ogura N., 1975. Further studies on decomposition of dissolved organic matter in coastal seawater, Mar. Biol., 31, 101-111.

Otsuki A. et Hanya T., 1972. Production of dissolved organic matter from dead green algae cells. I. Aerobic microbial decomposition. Limnol. Oceanogr., 17, 248-264.

PANSN (Port Autonome de Nantes St Nazaire), 1988. Campagne sur l'estuaire de la Loire, Fascicules de résultats.

Romaña L.A. et Thouvenin B., 1987. Faisabilité d'un modèle mathématique d'oxygene dissous dans l'estuaire de la Loire, Rapport interne IFREMER, DERO/EL 87.07, $85 \mathrm{p}$.

Saliot A. et le Groupe de Géochimie Organique du GRECO I.C.O., 1984. Biogéochimie de la matière organique en milieu estuarien : stratégies d'échantillonnage et de recherche élaborées en Loire (France), Oceanol. Acta, 7(2), 191, 207.

Salomon J.C., 1976. Modèle mathématique de la propagation de la marée en estuaire et des transports sableux associés. Application aux estuaires de la Loire et de la Seine. Thèse d'état, Université de Bretagne Occidentale.

Servais P., 1987. Etude la dégradation de la matiere organique par les bactéries hétérotrophes dans une rivière. Déve- loppement d'une démarche méthodologique et application à la Meuse belge. Thèse de $3^{e}$ cycle, Université Libre de Bruxelles, $271 \mathrm{p}$.

Sherr B. et Sherr E., 1983. Enumeration of heterotrophic microprotozoa by epifluorescence microscopy. Estuarine Coast. Shelf Sci., 16, 1-7.

Somville M., et Billen G., 1983. A method for determining exoproteolytic activity in natural waters. Limnol. Oceanogr., 28, 190-193.

Strickland J.D.H. et Parsons T.R., 1972. A practical handbook of seawater analysis. Bull. Fish. Res. Board Can., 167, $311 \mathrm{p}$.

Sumi T. et Koike I., 1990. Estimation of ammonification ammonium assimilation in surficial coastal and estuarine sediments. Limnol. Oceanogr., 35(2), 270-286.

Thouvenin B., Romaña L.A. et Le Hir P., 1992. Modélisation mathématique de l'oxygène dissous dans l'estuaire de la Loire, Rapport interne IFREMER, DEL/CCM/92.01.

Tuschall J.R. et Brezonik P.L., 1980. Characterization of organic nitrogen in natural waters: its molecular size, protein content an interaction with heavy metals. Limnol. Oceanogr., 25, 495504.

Usseglio-Polatera J.M. et Chenin-Mordojovick M.I., 1988. Fractionnal steps and process splitting methods for industrial codes. Proceedings of the VII International conference on Computational Methods in Waters Resources, Cambridge, USA.

Van Es F.B. et Laane W.P.M., 1982. The utility of organic matter in the Ems Dollard estuary. Neth. J. Sea Res., 1 : 101-118.

Van Wambecke F. et Bianchi M., 1986. Transfert et minéralisation de la matière organique azotée à travers les deux premiers échelons de la micro communaute hétérotrophe. Deuxieme Colloque International de Bactériologie 
Marine, CNRS, Brest, Oct. 1984, IFREMER, Actes de colloques, 3, 385391.

Wood E.D., Amstrong F.A.J. et Richards F.A., 1967. Determination of nitrate in sea water by cadmium copper reduction to nitrite. J. Mar. Biol. Ass. U.K., 47, 23-31.
Yoon W.B. et Rosson R.A., 1990. Improved method of enumeration of attached bacteria for study of fluctuation in the abundance of attached and free living bacteria in response to diel variation in seawater turbidity, Appl, and Env. Microbiol., Mar. 1990, Vol. 56, 3, 595-600. 